\title{
Commentary: Analysis of SUMO1-conjugation at synapses
}

\author{
Kevin A. Wilkinson ${ }^{1 *}$, Stéphane Martin ${ }^{2}$, Shiva K. Tyagarajan ${ }^{3}$, Ottavio Arancio ${ }^{4}$, \\ Tim J. Craig ${ }^{5}$, Chun Guo ${ }^{6}$, Paul E. Fraser ${ }^{7}$, Steven A. N. Goldstein ${ }^{8}$ and \\ Jeremy M. Henley ${ }^{1 *}$
}

\begin{abstract}
' School of Biochemistry, Centre for Synaptic Plasticity, University of Bristol, Bristol, United Kingdom, ${ }^{2}$ Centre National de la Recherche Scientifique, Institut National de la Santé et de la Recherche Médicale, IPMC, Université Côte d'Azur, Nice, France, ${ }^{3}$ Institute of Pharmacology and Toxicology, University of Zurich, Zurich, Switzerland, ${ }^{4}$ Taub Institute and Department of Pathology and Cell Biology, Columbia University, New York, NY, United States, ${ }^{5}$ Department of Applied Sciences, University of the West of England, Bristol, United Kingdom, ${ }^{6}$ Department of Biomedical Science, University of Sheffield, Sheffield, United Kingdom, ${ }^{7}$ Tanz Centre for Research in Neurodegenerative Diseases, University of Toronto, Toronto, ON, Canada, ${ }^{8}$ Stritch School of Medicine, Loyola University, Chicago, IL, United States
\end{abstract}

Keywords: SUMO1, synapse, sumoylation, neurons, post-translational modification

\section{A commentary on}

Analysis of SUMO1-conjugation at synapses

by Daniel, J. A., Cooper, B. H., Palvimo, J. J., Zhang, F. P., Brose, N., and Tirard, M. (2017). eLife 6:e26338. doi: 10.7554/eLife.26338

There is a large and growing literature on protein SUMOylation in neurons and other cell types. While there is a consensus that most protein SUMOylation occurs within the nucleus, SUMOylation of many classes of extranuclear proteins has been identified and, importantly, functionally validated. Notably, in neurons these include neurotransmitter receptors, transporters, sodium and potassium channels, mitochondrial proteins, and numerous key pre- and post-synaptic proteins (for reviews see Martin et al., 2007b; Scheschonka et al., 2007; Craig and Henley, 2012; Luo et al., 2013; Guo and Henley, 2014; Henley et al., 2014; Wasik and Filipek, 2014; Peng et al., 2016; Schorova and Martin, 2016; Wu et al., 2016). Furthermore, several groups have reported SUMO1-ylated proteins in synaptic fractions using biochemical subcellular fractionation approaches, using a range of different validated anti-SUMO1 antibodies (Martin et al., 2007a; Feligioni et al., 2009; Loriol et al., 2012; Luo et al., 2013; Marcelli et al., 2017) and many studies have independently observed colocalization of SUMO1 immunoreactivity with synaptic markers (Martin et al., 2007a; Konopacki et al., 2011; Gwizdek et al., 2013; Jaafari et al., 2013; Hasegawa et al., 2014; Ghosh et al., 2016). Tirard and co-workers (Daniel et al., 2017) directly challenge this wealth of compelling evidence. Primarily using a His6-HA-SUMO1 knock-in (KI) mouse, the authors contest any significant involvement of post-translational modification by SUMO1 in the function of synaptic proteins.

\section{ON WHAT BASIS DO DANIEL ET AL. ARGUE AGAINST SYNAPTIC SUMOYLATION?}

Accepted: 16 October 2017

Published: 30 October 2017

Citation:

Wikinson KA, Martin S, Tyagarajan SK, Arancio O, Craig TJ, Guo C, Fraser PE, Goldstein SAN and Henley JM (2017) Commentary: Analysis of SUMO1-conjugation at synapses.

Front. Cell. Neurosci. 11:345. Most of the experiments reported by Daniel et al. use a knock-in mouse that expresses His6-HA-SUMO1 in place of endogenous SUMO1. Using tissue from these mice, followed by immunoprecipitation experiments, they fail to biochemically identify SUMOylation of the previously validated SUMO targets synapsinla (Tang et al., 2015), gephyrin (Ghosh et al., 2016), GluK2 (Martin et al., 2007a; Konopacki et al., 2011; Chamberlain et al., 2012; Zhu et al., 2012), syntaxinla (Craig et al., 2015), RIM1a (Girach et al., 2013), mGluR7 (Wilkinson and Henley, 2011; Choi et al., 2016), and synaptotagmin1 (Matsuzaki et al., 2015). Moreover, by staining and subcellular fractionation, they also 
fail to detect protein SUMOylation in synaptic fractions or colocalization of specific anti-SUMO1 signal with synaptic markers. On this basis, they conclude there is essentially no functionally relevant SUMO1-ylation of synaptic proteins.

\section{WHAT ARE THE REASONS FOR THESE DISCREPANCIES?}

\section{Inefficiency of His6-HA-SUMO1 Conjugation and Compensation by SUMO-2/3}

A major cause for concern is that there is $20-30 \%$ less SUMO1-ylation in His6-HA-SUMO1 KI mice than in wildtype (WT) mice (Tirard et al., 2012; Daniel et al., 2017). Moreover, in the paper initially characterizing these KI mice, Tirard et al. showed that while total protein SUMO1-ylation is reduced, total SUMO2/3-ylation is correspondingly increased (Tirard et al., 2012). Thus, His6-HA-SUMO1 conjugation is significantly impaired and most likely compensated for by increased conjugation by SUMO2/3. Crucially, however, Daniel et al. do not examine modification by SUMO2/3 at any point in their recent study.

Given that SUMO modification is notoriously difficult to detect, the 20-30\% reduction in His6-HA-SUMO1 compared to wild-type SUMO1 conjugation will make it even more technically challenging. Moreover, this deficit in SUMO1-ylation may well be offset by an increase in SUMO2/3-ylation of individual proteins, but this likely compensation was not tested. Because these deficits alone could explain why Daniel et al. failed to detect SUMO1 modification of the previously characterized synaptic substrate proteins, it is surprising that they did not attempt to recapitulate the SUMO1-ylation of the target proteins under the endogenous conditions in wild-type systems used in the original papers since this approach would circumvent potential issues of ineffective conjugation or localization of the His6-HA-SUMO1.

\section{Lack of Functional Studies on the Substrates They Examine}

Daniel et al. confine their studies to immunoblotting and immunolabeling. However, these techniques address only one aspect of validating a bone fide SUMO substrate. It is at least as important to examine the effects of target protein SUMOylation in functional assays. Function-based approaches such as electrophysiology or neurotransmitter release assays are not reported or even discussed by Daniel et al. This is an extremely important omission. We argue that simply because SUMO1-ylation of a protein is beneath the detection sensitivity in a model system that exhibits sub-endogenous levels of SUMO1-ylation, does not mean that protein is not a functionally important and physiologically relevant SUMO1 substrate.

\section{Insensitivity or Inadequate Use of Assay Systems}

\section{Failure to Detect GluK2 SUMOylation}

GluK2 is a prototypic synaptic SUMO1 substrate that has been validated in exogenous expression systems, neuronal cultures, and rat brain (Martin et al., 2007a; Konopacki et al., 2011; Chamberlain et al., 2012; Zhu et al., 2012).

Daniel et al. attempt to detect SUMOylation of GluK2 using immunoprecipitation experiments from the His6-HA-SUMO1 KI mice. However, a key flaw in this experiment is that the C-terminal anti-GluK2 monoclonal rabbit antibody used does not recognize SUMOylated GluK2 because its epitope is masked by SUMO conjugation. Thus, due to technical reasons, the experiment shown could not possibly detect SUMOylated GluK2 whether or not it occurs in the KI mice.

\section{Subcellular Fractionation and Immunolabeling}

Daniel et al. perform subcellular fractionation and anti-SUMO1 Western blots to compare His6-HASUMO1 KI and SUMO1 knockout (KO) mice. In the KI mice they fail to detect SUMO1-ylated proteins in synaptic fractions. Importantly, however, they do not address what happens in WT mice, which, unlike the KI mice, exhibit normal levels of SUMO1-ylation.

While the authors provide beautiful images of SUMO1 immunolabeling in neurons cultured from WT, His6-HASUMO1 KI mice, and SUMO1 KO mice, in stark contrast to previous reports using rat cultures (Martin et al., 2007a; Konopacki et al., 2011; Gwizdek et al., 2013; Jaafari et al., 2013), they detect no specific synaptic SUMO1 immunoreactivity in neurons prepared from WT mice. We note, however, that the nuclear SUMO1 staining in neurons from His6-HA-SUMO1 KI mice is weak, and even weaker in WT neurons. Given that a very large proportion of SUMO1 staining is nuclear, these low detection levels would almost certainly rule out visualization of the far less abundant, but nonetheless functionally important, extranuclear SUMO1 immunoreactivity.

\section{IN CONCLUSION}

Given these caveats, we suggest that the failure of Daniel et al. to detect synaptic protein SUMO1-ylation in His6HA-SUMO1 KI mice is due to intrinsic deficiencies in this model system that prevent it from reporting the low, yet physiologically relevant, levels of synaptic protein modification by endogenous SUMO1. In consequence, we question the conclusions reached and the usefulness of this model for investigation of previously identified and novel SUMO1 substrates.

\section{AUTHOR CONTRIBUTIONS}

KW and JH wrote the first draft. All other authors contributed ideas, writing and creative input into the generation of the final manuscript.

\section{ACKNOWLEDGMENTS}

We are grateful to the Medical Research Council (MR/L003791/1 to KW and JH), Parkinson's UK (G-1605 to KW and $\mathrm{JH})$, the Biotechnology and Biological Sciences Research 
Council (BB/K014358 to TC and JH), the British Heart Foundation (PG/14/60/3101 to CG and JH), the Wellcome Trust (200581/Z/16/Z to TC), National Institutes of Health
(NIH-NS049442 to OA), and the Canadian Institutes of Health Research (TAD-117950 to PF) for financial support. We thank Dr. Ruth Carmichael for commenting on the manuscript.

\section{REFERENCES}

Chamberlain, S. E., Gonzalez-Gonzalez, I. M., Wilkinson, K. A., Konopacki, F. A., Kantamneni, S., Henley, J. M., et al. (2012). SUMOylation and phosphorylation of GluK2 regulate kainate receptor trafficking and synaptic plasticity. Nat. Neurosci. 15, 845-852. doi: 10.1038/ nn.3089

Choi, J. H., Park, J. Y., Park, S. P., Lee, H., Han, S., Park, K. H., et al. (2016). Regulation of mGluR7 trafficking by SUMOylation in neurons. Neuropharmacology 102, 229-235. doi: 10.1016/j.neuropharm.2015.11.021

Craig, T. J., Anderson, D., Evans, A. J., Girach, F., and Henley, J. M. (2015). SUMOylation of Syntaxin1A regulates presynaptic endocytosis. Sci. Rep. 5:17669. doi: $10.1038 /$ srep17669

Craig, T. J., and Henley, J. M. (2012). Protein SUMOylation in spine structure and function. Curr. Opin. Neurobiol. 22, 480-487. doi: 10.1016/j.conb.2011.10.017

Daniel, J. A., Cooper, B. H., Palvimo, J. J., Zhang, F. P., Brose, N., and Tirard, M. (2017). Analysis of SUMO1-conjugation at synapses. Elife 6:e26338. doi: 10.7554/eLife. 26338

Feligioni, M., Nishimune, A., and Henley, J. M. (2009). Protein SUMOylation modulates calcium influx and glutamate release from presynaptic terminals. Eur. J. Neurosci. 29, 1348-1356. doi: 10.1111/j.1460-9568.2009. 06692.x

Ghosh, H., Auguadri, L., Battaglia, S., Simone Thirouin, Z., Zemoura, K., Messner, S., et al. (2016). Several posttranslational modifications act in concert to regulate gephyrin scaffolding and GABAergic transmission. Nat. Commun. 7:13365. doi: $10.1038 /$ ncomms13365

Girach, F., Craig, T. J., Rocca, D. L., and Henley, J. M. (2013). RIM1 $\alpha$ SUMOylation is required for fast synaptic vesicle exocytosis. Cell Rep. 5, 1294-1301. doi: $10.1016 /$ j.celrep.2013.10.039

Guo, C., and Henley, J. M. (2014). Wrestling with stress: roles of protein SUMOylation and deSUMOylation in cell stress response. IUBMB Life 66, 71-77. doi: 10.1002/iub.1244

Gwizdek, C., Casse, F., and Martin, S. (2013). Protein sumoylation in brain development, neuronal morphology and spinogenesis. Neuromolecular Med. 15, 677-691. doi: 10.1007/s12017-013-8252-Z

Hasegawa, Y., Yoshida, D., Nakamura, Y., and Sakakibara, S. (2014). Spatiotemporal distribution of SUMOylation components during mouse brain development. J. Comp. Neurol. 522, 3020-3036. doi: 10.1002/cne. 23563

Henley, J. M., Craig, T. J., and Wilkinson, K. A. (2014). Neuronal SUMOylation: mechanisms, physiology, and roles in neuronal dysfunction. Physiol. Rev. 94, 1249-1285. doi: 10.1152/physrev.00008.2014

Jaafari, N., Konopacki, F. A., Owen, T. F., Kantamneni, S., Rubin, P., Craig, T. J., et al. (2013). SUMOylation is required for glycine-induced increases in AMPA receptor surface expression (ChemLTP) in hippocampal neurons. PLoS ONE 8:e52345. doi: 10.1371/journal.pone.0052345

Konopacki, F. A., Jaafari, N., Rocca, D. L., Wilkinson, K. A., Chamberlain, S., Rubin, P., et al. (2011). Agonist-induced PKC phosphorylation regulates GluK2 SUMOylation and kainate receptor endocytosis. Proc. Natl. Acad. Sci. U.S.A. 108, 19772-19777. doi: 10.1073/pnas.1111575108

Loriol, C., Parisot, J., Poupon, G., Gwizdek, C., and Martin, S. (2012). Developmental regulation and spatiotemporal redistribution of the sumoylation machinery in the rat central nervous system. PLoS ONE 7:e33757. doi: 10.1371/journal.pone.0033757

Luo, J., Ashikaga, E., Rubin, P. P., Heimann, M. J., Hildick, K. L., Bishop, P., et al. (2013). Receptor trafficking and the regulation of synaptic plasticity by SUMO. Neuromolecular Med. 15, 692-706. doi: 10.1007/s12017-013-8253-y

Marcelli, S., Ficulle, E., Iannuzzi, F., Kovari, E., Nistico, R., and Feligioni, M. (2017). Targeting SUMO-1ylation contrasts synaptic dysfunction in a mouse model of Alzheimer's disease. Mol. Neurobiol. 54, 6609-6623. doi: 10.1007/s12035-016-0176-9

Martin, S., Nishimune, A., Mellor, J. R., and Henley, J. M. (2007a). SUMOylation regulates kainate-receptor-mediated synaptic transmission. Nature 447, 321-325. doi: 10.1038/nature05736

Martin, S., Wilkinson, K. A., Nishimune, A., and Henley, J. M. (2007b). Emerging extranuclear roles of protein SUMOylation in neuronal function and dysfunction. Nat. Rev. Neurosci. 8, 948-959. doi: 10.1038/nrn2276

Matsuzaki, S., Lee, L., Knock, E., Srikumar, T., Sakurai, M., Hazrati, L. N., et al. (2015). SUMO1 affects synaptic function, spine density and memory. Sci. Rep. 5:10730. doi: 10.1038/srep 10730

Peng, J., Ren, K. D., Yang, J., and Luo, X. J. (2016). Mitochondrial E3 ubiquitin ligase 1: a key enzyme in regulation of mitochondrial dynamics and functions. Mitochondrion 28, 49-53. doi: 10.1016/j.mito.2016.03.007

Scheschonka, A., Tang, Z., and Betz, H. (2007). Sumoylation in neurons: nuclear and synaptic roles? Trends Neurosci. 30, 85-91. doi: 10.1016/j.tins.2007.01.003

Schorova, L., and Martin, S. (2016). Sumoylation in synaptic function and dysfunction. Front. Synaptic Neurosci. 8:9. doi: 10.3389/fnsyn.2016.00009

Tang, L. T., Craig, T. J., and Henley, J. M. (2015). SUMOylation of synapsin Ia maintains synaptic vesicle availability and is reduced in an autism mutation. Nat. Commun. 6:7728. doi: 10.1038/ncomms 8728

Tirard, M., Hsiao, H. H., Nikolov, M., Urlaub, H., Melchior, F., and Brose, N. (2012). In vivo localization and identification of SUMOylated proteins in the brain of His6-HA-SUMO1 knock-in mice. Proc. Natl. Acad. Sci. U.S.A. 109, 21122-21127. doi: 10.1073/pnas.1215366110

Wasik, U., and Filipek, A. (2014). Non-nuclear function of sumoylated proteins. Biochim. Biophys. Acta 1843, 2878-2885. doi: 10.1016/j.bbamcr.2014.07.018

Wilkinson, K. A., and Henley, J. M. (2011). Analysis of metabotropic glutamate receptor 7 as a potential substrate for SUMOylation. Neurosci. Lett. 491, 181-186. doi: 10.1016/j.neulet.2011.01.032

Wu, H., Chen, X., Cheng, J., and Qi, Y. (2016). SUMOylation and potassium channels: links to epilepsy and sudden death. Adv. Protein Chem. Struct. Biol. 103, 295-321. doi: 10.1016/bs.apcsb.2015.11.009

Zhu, Q. J., Xu, Y., Du, C. P., and Hou, X. Y. (2012). SUMOylation of the kainate receptor subunit GluK2 contributes to the activation of the MLK3JNK3 pathway following kainate stimulation. FEBS Lett. 586, 1259-1264. doi: 10.1016/j.febslet.2012.03.048

Conflict of Interest Statement: The authors declare that the research was conducted in the absence of any commercial or financial relationships that could be construed as a potential conflict of interest.

Copyright (C) 2017 Wilkinson, Martin, Tyagarajan, Arancio, Craig, Guo, Fraser, Goldstein and Henley. This is an open-access article distributed under the terms of the Creative Commons Attribution License (CC BY). The use, distribution or reproduction in other forums is permitted, provided the original author(s) or licensor are credited and that the original publication in this journal is cited, in accordance with accepted academic practice. No use, distribution or reproduction is permitted which does not comply with these terms. 